\title{
BRONCHOSCOPIC MANAGEMENT OF CENTRAL AIRWAY OBSTRUCTION
}

Kenton E. Stephens, Jr, MD

Douglas E. Wood, MD
Objectives: Patients with central airway obstruction are critically ill, with impending suffocation. They are seen with diverse anatomic and functional deficits caused by both benign and malignant obstructions. Such cases were reviewed to examine the indications, techniques, and outcomes of an algorithm approach to bronchoscopic management. Methods: Between July 1992 and April 1996, 97 patients underwent bronchoscopic procedures for the management of central airway obstruction, and their cases were used for a retrospective review of the airway management. Results: There were 48 male and 49 female patients, aged 13 to 85 years. There were 48 benign and 49 malignant pathologic conditions that gave rise to 108 stenoses. These were treated with 199 endoscopic procedures with an average of 1.7 interventions per endoscopy, including mechanical core-out (62), dilation (135), laser ablation (44), placement of brachytherapy catheters (9), and stent placement (88). Diagnoses included lung cancer, primary tracheobronchial tumors, tumors metastatic to the airway or mediastinum, and a variety of benign obstructions. In the group of 97 patients there were $2(2 \%)$ perioperative deaths and $34(34 \%)$ late deaths, 29 in the malignant group and 5 in the benign group. Median survival was 7.6 months (range 1 week-31 months). There were $7(7 \%)$ complications among the group of 97,4 in the malignant group, and 3 in the benign group. Conclusions: Endobronchial surgical techniques can be used safely and systematically for the relief of benign and malignant central airway obstructions; a diversity of approaches and interventions are required to produce and maintain palliation of airway symptoms. (J Thorac Cardiovasc Surg 2000; 119:289-96)
$\mathrm{C}^{\mathrm{s}}$ entral airway obstruction produces symptoms of dyspnea, stridor, and obstructive pneumonia and is frequently life-threatening, with patients having impending suffocation. In most cases, resection and surgical reconstruction provides the best opportunity for definitive management. However, bronchoscopic management is the first step in providing a diagnosis, stabilizing the obstructed airway, and evaluating

From the Section of General Thoracic Surgery, University of Washington, Seattle, Wash.

Read at the Twenty-second Annual Meeting of The Western Thoracic Surgical Association, Maui, Hawaii, June 26-29, 1996.

Received for publication Feb 15, 1999; revisions requested April 30, 1999; revisions received Sept 22, 1999; accepted for publication Sept 23, 1999.

Address for reprints: Douglas E. Wood, MD, University of Washington, Box 356310, 1959 NE Pacific, Room AA-115, Seattle, WA 98195.

Copyright (C) 2000 by Mosby, Inc.

$0022-5223 / 2000 \$ 12.00+0 \quad \mathbf{1 2 / 6 / 1 0 3 3 0 7}$ resectability. In patients in whom the obstruction is unresectable because of anatomic limitations, metastatic disease, or overall medical condition, endoscopic techniques are minimally invasive and can provide significant palliation. Although the long-term outlook in these cases is often dismal, the temporary or permanent relief of airway obstruction provides significant palliation with marked improvement in quality of life and, in many cases, prolongation of life.

The essential principles of endoscopic management of malignant airway obstruction have been outlined concisely by Mathisen and Grillo. ${ }^{1}$ Advances in airway prosthetics have provided a variety of silicone stents, expandable metal stents, and pneumatic dilators, enabling the surgeon to correct increasingly complex anatomic problems. Several series have been published describing the application and results of these techniques. $^{1-4}$

Because of the relative rarity of airway stenoses and the infrequent consideration of surgical or endoscopic 
palliation by primary or specialty physicians, it is uncommon to see a significant number of these problems in a thoracic surgical practice. With the establishment of an endoscopic and surgical airway practice at the University of Washington in 1992, the Thoracic Surgical Service has received an increasing number of referrals for the management of central airway obstruction. We have reviewed our experience in a consecutive series of 97 patients treated with therapeutic bronchoscopy to delineate the etiology of central airway obstruction and results of airway therapeutics.

\section{Patients and methods}

Patients. The patient group consisted of 97 consecutive patients seen with symptomatic central airway obstruction between July 1992 and April 1996. Patients were included if they underwent bronchoscopic treatment for obstruction of the central airways, defined as the trachea, carina, right or left main-stem bronchus, or the bronchus intermedius. Interventions included dilation, core-out of tumor, laser ablation, stenting, and high-dose intraluminal radiation (brachytherapy). Diagnostic examination with a flexible or rigid bronchoscope without therapeutic interventions was excluded. All procedures were done at the University of Washington Medical Center in Seattle.

Preoperative management. Prereferral radiologic studies (chest radiograph and chest computed tomography) along with a careful history and physical examination and the referring physician's bronchoscopy report were reviewed to determine which symptoms were attributable to airflow obstruction versus secondary pathologic conditions such as atelectasis or pneumonia. Flexible bronchoscopes were avoided in the evaluation of critically narrowed airways because of the potential for occlusion of the remaining lumen, with no ability to control the airway or to ventilate the lung through the bronchoscope. Patients with severe stridor had institution of medical measures for temporary stabilization of the condition, with inspired cool saline mist, nebulized racemic epinephrine, mild sedation, and systemic steroids.

Anesthetic technique. The surgeon was at the bedside for the entire induction sequence and was prepared for emergency airway control with the rigid bronchoscope. Careful preoperative planning and intraoperative communication between the surgeon and anesthesiologist were emphasized, along with training of a dedicated team of nurses and anesthesiologists familiar with the problems and equipment used for endoscopic airway management. Anesthetic induction maintained spontaneous ventilation with the use of a combination of inhaled or short-acting intravenous agents until a secure airway could be established. ${ }^{5}$ In cases in which the $\mathrm{Nd}$ :YAG laser was used, the inspired oxygen fraction was maintained at less than $50 \%$ to avoid combustion within the airway.

Bronchoscopy. Rigid bronchoscopy was done with a Storz (Shapshay laser broncho-tracheoscope, Karl Storz Endoscopy
America, Inc, Culver City, Calif) or Jackson (Pilling-Weck, Research Triangle, NC) bronchoscope. Appropriately sized Jackson bronchoscopes $(3.5,4,5,6,7,8$, and $9 \mathrm{~mm})$ were used for initial dilation and airway control. The Jackson bronchoscopes have the advantage of a blunt, rounded tip, making it easier to use them for dilation with less risk of perforation or mucosal laceration. The Storz bronchoscope has no internal light carrier, a smooth inner lumen, and multichanneled attachments, ideal for use of the laser and for placement of smaller $(<14 \mathrm{~mm})$ stents through the lumen of the bronchoscope. Endoscopic magnification was provided by a telescope (Hopkins straight-forward telescope; Karl Storz Endoscopy America) attached to a video monitor to provide visualization for the whole surgical team and to aid in resident education. Measurements of the length of the lesion were recorded, as well as its relation to the vocal cords, cricoid, carina, and lobar orifices.

Dilation. Dilation was used primarily for benign airway stenosis. After inspection and measurement, serial dilation was done with successively larger bronchoscopes. Steady rotating pressure with the blunt-tipped Jackson bronchoscopes provided passage with minimal mucosal trauma and risk of perforation. The result was inspected, and the procedure was repeated with the next larger bronchoscope until an adequate airway caliber had been established. If the lesion was too stenotic to accept the 3.5-mm Jackson bronchoscope, then esophageal bougies (Jackson flexible, Karl Storz Endoscopy America) were used to enlarge the airway enough to allow bronchoscopic dilation. Pneumatic or hydrostatic balloon dilation with angioplasty balloons was used whenever it was necessary to dilate larger than the 8 to $9 \mathrm{~mm}$ possible with bronchoscopic dilation. ${ }^{6}$

Core-out. In malignant lesions the intraluminal mass was traversed following the most normal-appearing bronchial wall opposite the mass. In the initial examination, intentional coring out of the tumor was not usually the objective, because of the unknown distal anatomy. Instead, the bronchoscope was used to press the obstruction onto its pedicle, establishing control of the airway and allowing evaluation of the extent of the pathologic condition. A mechanical core-out of tumor was then done by engaging the endobronchial tumor with the tip of the rigid bronchoscope and by debridement with biopsy forceps.

Laser ablation. Laser vaporization was done predominately for residual endobronchial tumor after mechanical coreout, for tumors inaccessible to mechanical debridement, and occasionally for airway granulations. An Nd:YAG laser (MBB-AT Medilas 2 Nd:YAG 621, MBB-Angewandte, Munich, Germany) was used at a power setting of 35 watts and 0.5 -second pulse duration.

Stents. Stenting techniques were similar to those previously described in the literature..$^{1-3,7-11}$ Indications for stenting included (1) recurrent benign strictures that were not surgically resectable, (2) active inflammation or edema necessitating acute and possibly temporary support while the pathologic process resolved, (3) rapidly growing or recurrent 
obstructive endobronchial tumors, (4) tumors producing extrinsic obstruction, (5) early anastomotic strictures that might stabilize over a stent during postoperative remodeling, and (6) tracheobronchial malacia. Because stents are palliative and frequently necessitate ongoing management, they were used only after excluding definitive surgical correction or other, more simple, endoscopic palliation.

For simple benign and malignant stenoses the stent of choice was a simple flanged or studded silicone stent (Hood Laboratories, Pembroke, Mass). If the carina was involved, a silicone Y stent was placed with a proximal tracheal limb and bilateral main-stem bronchial limbs. If the orifice of the right upper lobe was obstructed by a stent in the right main-stem bronchus, it was removed and the distal end beveled slightly before it was replaced and rechecked for lobar patency. In cases in which the predominant pathologic condition was tracheobronchial malacia the Montgomery T tube was used for upper tracheal lesions ${ }^{2}$ and carinal Y stents for distal lesions ${ }^{11}$ (Hood Laboratories). These have the advantage of stabilization by the T-limb through a tracheal stoma or the Y-shaped geometry of the carina, respectively. Expandable metal stents (Schneider Wallstent, Medinvent SA, Lausanne, Switzerland) were also used in the setting of malacia because these stents expand and ultimately imbed within the airway, largely preventing migration. ${ }^{12,13}$ Expandable stents were also used occasionally in the setting of difficult anatomy in which seating of the Wallstent expandable stent was more secure than that of other stents or it provided a significantly better luminal diameter (especially for small-caliber bronchi).

Silicone stents larger than $14 \mathrm{~mm}$ were delivered by prepositioning a pusher tube and the stent over an appropriately sized bronchoscope. Stents $14 \mathrm{~mm}$ and smaller were partially folded and introduced through the 10-mm Storz bronchoscope. For Y stents, extra manipulations were necessary to ensure that both limbs unfolded into the main-stem bronchi. This was ensured by guiding 1 limb over a prepositioned balloon catheter ${ }^{3}$ or by manipulating the carina of the stent with a forceps. ${ }^{14}$ Wallstent and Permalume stents were deployed from a flexible sheath, which permitted deployment under combined endoscopic and fluoroscopic control.

Brachytherapy. High-dose endobronchial radiation was used as an adjuvant therapy to core-out and stenting procedures in cases with a dominant component of obstructing endobronchial tumor. Flexible or rigid bronchoscopy guided placement of a polyethylene afterloading catheter or catheters into the involved airway, followed by placement of multiple iridium-192 seeds with 1-cm spacing, calculated to produce a dose of 1000 to $1500 \mathrm{cGy}$ at $1-\mathrm{cm}$ diameter from the catheter along the course of the endobronchial tumor.

Treatment algorithm. Rigid bronchoscopy was used in all patients with symptomatic central airway obstruction to establish a controlled airway and to evaluate the location and extent of pathologic conditions. Patients with resectable disease, benign or malignant, were treated by airway resection and reconstruction. Patients with unresectable conditions were treated according to a systematic but flexible algorithm as shown in Fig 1. Patients with benign stenosis were treated

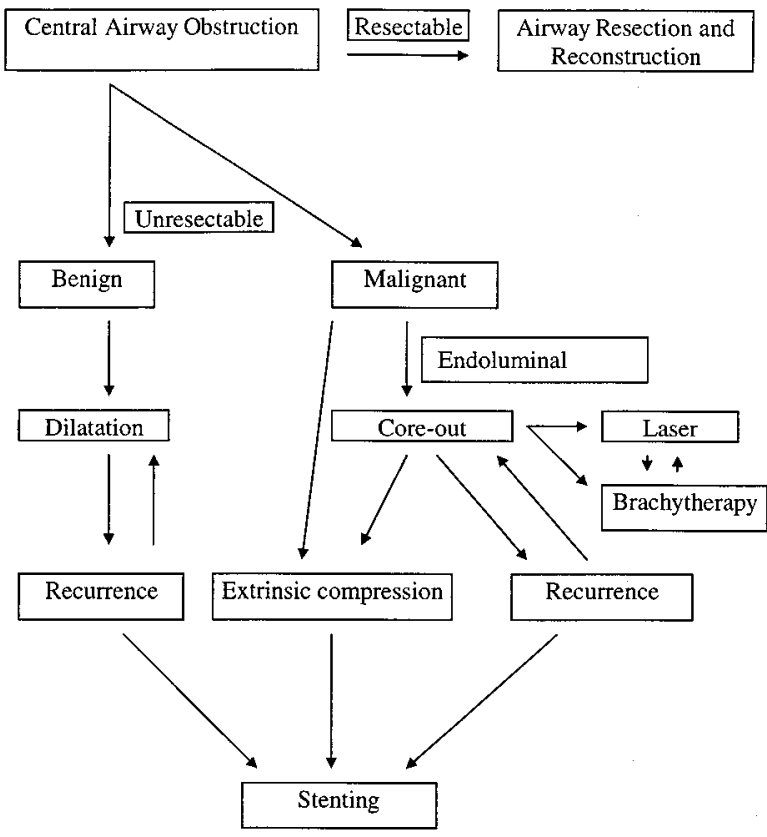

Fig 1. Algorithm for the endoscopic management of central airway obstruction.

initially by dilation, with stents placed for rapidly recurrent or recalcitrant strictures. Malignant obstruction was managed primarily by a core-out of the tumor, with the use of the $\mathrm{Nd}$ :YAG laser and brachytherapy as adjuncts to mechanical débridement. Stents were placed for recurrent endobronchial tumors or significant extrinsic compression.

Patient follow-up. Hospital records were reviewed retrospectively to document etiology and location of the obstructive lesions and the interventions used and to detect hospital and outpatient deaths. Surviving patients were sought by telephone inquiry. If this failed, inquiries to the referring physician were made.

\section{Results}

Patient demographics. Ninety-seven consecutive patients underwent therapeutic bronchoscopy between July 1992 and April 1996. There were 48 male and 49 female patients, aged 13 to 85 years. There were 20 male and 28 female patients with benign diagnoses, aged 13 to 80 years ( $49 \pm 17.4$ years). In the malignant group, there were 28 male and 21 female patients with ages from 27 to 85 years $(58.7 \pm 12.6$ years). A total of 108 stenoses were treated in the 97 patients ( 56 benign, 52 malignant).

Location and pathology. The locations of the lesions treated in this study are shown in Table I. Benign lesions more commonly involved the proximal trachea and mid trachea with only two benign stenoses of the distal trachea and an even distribution through- 
Table I. Location of lesions

\begin{tabular}{lccc}
\hline Location & Benign & Malignant & Total \\
\hline Proximal trachea & 18 & 3 & 21 \\
Mid trachea & 9 & 5 & 14 \\
Distal & 2 & 5 & 7 \\
Left main stem & 12 & 8 & 20 \\
Right main stem & 6 & 9 & 15 \\
Bronchus intermedius & 8 & 16 & 24 \\
Lobar bronchi & 1 & 6 & 7 \\
Total & 56 & 52 & 108 \\
\hline
\end{tabular}

Table II. Etiology of benign lesions

\begin{tabular}{lc}
\hline Etiology & No. \\
\hline Anastomotic & 14 \\
Tracheostomy & 9 \\
Tracheal intubation & 7 \\
Vascular compression & 4 \\
Tracheomalacia & 4 \\
Idiopathic & 4 \\
Other & 6 \\
Total & 48 \\
\hline
\end{tabular}

Table III. Etiology of malignant lesions

\begin{tabular}{lrl}
\hline Etiology & No. \\
\hline Primary tracheobronchial or lung tumors & & \\
Non-small cell lung cancer & 20 & \\
Small cell lung cancer & 1 & \\
Primary tracheal tumor & 4 & \\
Bronchial carcinoid & 27 \\
Total & & 27 \\
Distant primary tumors metastatic to trachea & & \\
or mediastinum & 8 & \\
Renal cell & 5 & \\
Thyroid & 3 & \\
Metastatic sarcoma & 1 & \\
Carcinoid & 1 & \\
Cervix & 1 & \\
Colon & 1 & \\
Peripheral neuroectodermal tumor & 1 & \\
Prostate & 1 & \\
Head and neck & & 22 \\
Total & & 49 \\
Total & &
\end{tabular}

out the main-stem bronchi and bronchus intermedius. The etiology of the benign lesions is tabulated in Table II. The majority of benign lesions were the sequelae of tracheostomy, prolonged intubation, or airway anastomosis, and there were a number of miscellaneous causes. Four patients had major airway obstruction caused by massive pulmonary artery dilation as a result of con-
Table IV. Distribution of interventions, excluding stents

\begin{tabular}{lccr}
\hline Procedure & Benign & Malignant & Total \\
\hline Core-out & 19 & 43 & 62 \\
Bronchoscopic dilation & 68 & 43 & 111 \\
Balloon dilation & 5 & 0 & 5 \\
Bougie dilation & 17 & 2 & 19 \\
Brachytherapy & 0 & 9 & 9 \\
Laser ablation & 11 & 33 & 44 \\
\hline
\end{tabular}

Table V. Stent use

\begin{tabular}{lccc}
\hline Type of stent & Benign & Malignant & Total \\
\hline Simple & 46 & 25 & 71 \\
T, Y, or tracheostomy & 9 & 2 & 11 \\
Expandable metal & 1 & 5 & 6 \\
\hline
\end{tabular}

genital heart disease. Another distinct group of patients comprised the 4 who had malacia of the trachea or bronchus. The endoscopic findings in these cases included a loss of structural rigidity resulting in dynamic inspiratory collapse. The precise cause was known in only 1 of these cases, and this was relapsing polychondritis.

The etiology of the malignant lesions is tabulated in Table III. Fifty-five percent (27/49) of these lesions were a result of primary tracheobronchial or lung tumors. The majority of these were caused by central extension of bronchogenic carcinoma. Four patients had obstructive tracheal tumors, which consisted of 1 squamous, 1 mucoepidermoid, and 2 adenoid cystic tumors. Forty-five percent (22/49) of malignant lesions were a result of metastatic disease to the airway or mediastinum and these consisted mostly of renal cell carcinoma, thyroid carcinoma, and metastatic sarcoma.

Distribution of procedures. A therapeutic bronchoscopy procedure was defined as a single trip to the operating room for treatment of an obstructing lesion. An intervention was defined as a discrete operative technique applied to a lesion. For example, if a malignant lesion was cored out for treatment of an endoluminal mass and then stented for residual extrinsic compression, this would represent 1 procedure consisting of 2 discrete interventions. The 97 patients underwent a total of 199 procedures with 338 interventions. Of these 338 procedures, 250 were endoscopic manipulations of the airway (Table IV) and 88 were stent placement, removal, or revision.

Stents. Seventy-one of the stents placed were simple 
flanged or studded silicone stents. Eleven stents were of a more complex design such as a $\mathrm{T}$ or $\mathrm{Y}$ configuration. The distribution between groups is shown in Table $\mathrm{V}$. In the benign group, $3 \mathrm{Y}$ stents, $4 \mathrm{~T}$ tubes, and 2 tracheostomy appliances were used. In the malignant group, 2 Y stents were used, not for carinal or bilateral involvement, but because of proximal unilateral mainstem bronchus involvement where simple stent placement appeared insecure. Expandable stents were used in 6 patients, mostly in cases of malignant disease in which the anatomy interfered with adequate seating or patency of the silicone stents.

Palliation of obstruction. Successful palliation was achieved in 91 (94\%) of 97 patients. This required multiple procedures in 41 patients and more than 1 intervention per procedure in many patients to obtain or maintain the desired result. It was not possible to palliate the airway obstruction in 6 patients because of impassable obliteration of the lumen or distal extension into lobar or segmental bronchi.

Deaths and complications. Two deaths (2\%) occurred during the same hospital admission or within 30 days of the procedure. No deaths occurred intraoperatively. There were 34 known late deaths. Of the 16 patients with a known date of death, the survival after airway intervention ranged from 1 week to 31 months, with a mean of 7.6 months. All but 5 of the deaths were in the malignant group. Two patients with airway compromise from pulmonary artery dilation who received silicone stents died of uncontrollable airway hemorrhage 40 and 45 days after operation, respectively. Autopsies failed to reveal perforation of the stent into the pulmonary artery. Of the 3 remaining patients with benign disease who died, 2 were lung transplant recipients who died of infectious complications and 1 was a patient treated for a bronchial stenosis who died of complications of coronary artery bypass grafting. No deaths were attributable to obstruction of indwelling stents.

Assignment of complications is somewhat difficult in a therapeutic modality in which transient palliation is commonly the objective. Overall, there were 7 complications $(7 \%)$. In the malignant group there were 4 complications: 3 stent dislodgements necessitating replacement and 1 stent occlusion by tumor. In the benign group there were 3 complications: 2 stent migrations necessitating repositioning and 1 stent compression necessitating replacement with a stiffer stent.

\section{Discussion}

This series represents a single-institution experience with the bronchoscopic management of central airway obstruction. There are two similar series describing the cases of patients undergoing complex bronchoscopic management of central airway obstruction with use of a variety of techniques. ${ }^{1,3}$ Mathisen and Grillo ${ }^{1}$ reported on 56 patients with central airway obstruction caused by neoplasms, and the series of Sonett and colleagues ${ }^{3}$ reported on 57 patients with central airway obstruction with a variety of etiologies. Both of these reports emphasized the importance of a simple but flexible and diverse approach to these lesions, as well as the satisfying results possible from palliation of an obstructed airway. Other reports have focused on a single modality therapy such as laser ablation or stenting.,10,11,14-16 However, this is a heterogeneous patient population with wide variability in the severity of the condition, etiology, and anatomic obstacles producing airway compromise. Our goal has been to approach these cases with a diversity of techniques as part of a dedicated tracheobronchial surgical program. This series of 97 consecutive patients achieves similar results to those of earlier series, emphasizes the diverse pathology of these lesions, and corroborates our clinical impression that patients receive significant symptomatic relief from a systematic approach to these problems.

Bronchoscopic management of airway obstruction is almost always palliative and should only be considered when a definitive approach is not feasible. Because it is a palliative procedure, repeated interventions often are required to maintain airway patency. Forty-one patients $(42 \%)$ required more than 1 procedure to obtain or maintain airway patency, with some patients requiring as many as 15 separate procedures. Analysis of the pathologic conditions involved further supports the palliative nature of most endobronchial interventions. Benign postintubation, anastomotic, and postinflammatory stenoses result in a full-thickness fibrous scar. Although dilation or laser "resection" might produce a temporary improvement in airway symptoms, these treatments usually produce further inflammation with recurrent stenosis that, after repeated procedures, might become more severe than the original lesion. Stenting for benign or malignant lesions is clearly without curative intent and requires long-term management to correct potential stent problems, such as migration or obstruction by inspissated secretions, granulation tissue, or tumor. Stents might also erode into surrounding structures, with possible exsanguinating hemorrhage from bronchovascular fistulas. ${ }^{17}$ We encountered 2 cases of fatal hemorrhage after placement of silicone stents. Both occurred in the setting of severe pulmonary hypertension. There was no autopsy evidence of a bronchopulmonary artery fistula or stent erosion in these cases, which were both diagnosed as hemoptysis 
as a result of pulmonary hypertension. However, we remain cautious about the use of stents for vascular airway compression because of the concern regarding fistula formation and fatal airway hemorrhage. ${ }^{17}$

Although airway resection and reconstruction is the preferred therapy for both benign and malignant lesions, ${ }^{18,19}$ a variety of factors including a long stenosis, failed previous repair, metastatic or unresectable malignancy, or patient refusal might dictate nonsurgical management. In other cases, endoscopic management might act as temporizing palliation while the patient's condition is stabilized or evaluated for subsequent resection. Experience with interventional bronchoscopy is an important part of any thoracic surgical program that performs airway resections or lung transplantation, providing for management of postoperative anastomotic complications. The surgeon or pulmonologist considering bronchoscopic palliation should likewise be experienced with both the indications and techniques of airway resection or be a part of a closely linked multidisciplinary team that allows a balanced consideration of airway resection versus therapeutic bronchoscopy.

The algorithm used in this series tackles each unique problem-pedunculated tumor, scar, external airway compression, or circumferential tumor-with a customized set of techniques. Malignant endoluminal obstruction is cored out and the base or difficult-toreach remnants vaporized by the Nd:YAG laser. Adjuvant brachytherapy is used to prolong patency in cases of predominant endobronchial tumor or limited extrabronchial disease. The effective dose diameter of endobronchial radiation is 0.5 to $2.0 \mathrm{~cm}$ and so is not effective treatment for bulky tumors or those producing extrinsic compression. ${ }^{20}$ If the endobronchial disease has been or appears likely to be rapidly recurrent, or if significant endobronchial compression remains, an endobronchial stent is placed. In most cases, a solid silicone stent can be seated securely, which has the combined advantages of being inexpensive and being easily repositioned, replaced, or removed. This is particularly important if the indications for stenting are possibly temporary, as in malignant disease during therapy and in benign disease while short-term remodeling or resolution of inflammation occur. Expandable wire mesh stents are chosen when it is not possible to adequately seat a silicone stent or in cases with severe external compression that causes distortion and persistent obstruction of a silicone stent. When expandable stents are chosen, the silicone-covered stents are preferred to avoid tumor ingrowth or granulations through the interstices of the stent. Photodynamic therapy
(PDT) has been used in some centers for malignant airway obstruction and involves a photosensitizing agent that, when exposed to light in the proper wavelength, forms toxic oxygen radicals that result in cell death. PDT is not widely available, in contrast to the other techniques described for airway palliation, and its use in the lung has been described primarily for the treatment of superficial squamous carcinomas involving the central airways. ${ }^{21}$ However, PDT has also been used in several centers for the treatment of obstructing tracheobronchial tumors and has been found to have the best response in polypoid tumors with a poor response in submucosal or peribronchial disease. ${ }^{22}$ Although PDT might prove to have a significant therapeutic role in certain superficial inoperable lung cancers, it does not appear to provide any significant advantages in the palliation of obstructing lesions over the techniques of core-out, laser ablation, and stenting, which avoid the consequences of photosensitivity in patients undergoing PDT.

Benign strictures are dilated with esophageal bougies, the bronchoscope, or appropriately sized angioplasty balloons. Recurrent unresectable benign stricture is an indication for endoluminal stenting. Lesions that extend proximally into the subglottic larynx usually necessitate use of a silicone stent with an external limb ( $\mathrm{T}$ tube) to allow stabilization of the subglottic or supraglottic stent. Expandable stents are relatively contraindicated in this position because of their permanence and the difficulties with proximal subglottic granulations that might extend the original obstruction. In cases of benign tracheobronchial malacia, silicone stents are difficult to seat adequately and in these patients stabilization of the stent by an external limb (T tube), by the geometry of a carinal Y stent, or by placement of an expandable stent may be required.

Although the skills and procedures described for therapeutic bronchoscopy are not particularly difficult, application of these skills in the patient with a severely compromised airway is challenging, even for physicians with significant experience in airway therapeutics. Because of the paramount importance of the airway, the learning curve can be hazardous, especially in unstable situations. Successful procedures rely heavily on both volume and an experienced team of anesthesiologists and nurses. This systematic approach also aids in the instruction of thoracic surgical residents.

Bronchoscopic therapeutics are safe and effective when applied to central airway obstructions from a variety of causes. In only 6 instances in this series was the objective of restoring airway patency technically impossible. This was due to either obliteration of the 
lumen by impenetrable postsurgical scar or diffuse stenosis extending into the lobar and segmental bronchi. The insurmountable problem was identified in each case and the procedure terminated without technical misadventure. In the remaining cases, initial improvement was achieved anatomically and, with few exceptions, symptomatically.

\section{REFERENCES}

1. Mathisen DJ, Grillo HC. Endoscopic relief of malignant airway obstruction. Ann Thorac Surg 1989;48:469-75.

2. Cooper JD, Pearson FG, Patterson GA, Todd TRJ, Ginsberg RJ, Goldberg M, et al. Use of silicone stents in the management of airway problems. Ann Thorac Surg 1989;47:371-8.

3. Sonett JR, Keenan RJ, Ferson PF, Griffith BP, Landreneau RJ. Endobronchial management of benign, malignant and lung transplantation airway stenoses. Ann Thorac Surg 1995;59:1417-22.

4. Petrou M, Kaplan D, Goldstraw P. Bronchoscopic diathermy resection and stent insertion: a cost effective treatment for tracheobronchial obstruction. Thorax 1993;48:1156-9.

5. Wilson RS. Anesthetic management for tracheal reconstruction: international trends in general thoracic surgery. Vol 2. Philadelphia: WB Saunders; 1987. p. 3-12.

6. Carlin BW, Harrell JH, Moser KM. The treatment of endobronchial stenosis using balloon catheter dilatation. Chest 1988;93:1148-51.

7. Colt HG, Dumon JF. Airway stents: present and future. Clin Chest Med 1995;16:465-78.

8. Mehta AC, Harris RJ, DeBoer GE. Endoscopic management of benign airway stenoses. Clin Chest Med 1995;16:401-13.

9. Gaer JAR, Tsang V, Khanghani A, et al. Use of endotracheal silicone stents for relief of tracheobronchial obstruction. Ann Thorac Surg 1992;54:512-6.

10. Bollinger CT, Probst R, Tschopp K, Soler M, Perruchoud AP. Silicone stents in the management of inoperable tracheobronchial stenosis. Chest 1993;104:1653-9.

11. Westaby S, Jackson JW, Pearson FG. A bifurcated silicone rubber stent for relief of tracheobronchial obstruction. J Thorac Cardiovasc Surg 1982;83:414-7.

12. Kind CRK, Donnelly RJ. Expandable metal stents for tracheal obstruction: permanent or temporary? A cautionary tale. Thorax 1992;47:757-8.

13. Nashef SAM, Dromer C, Velly JF, Labrousse L, Couraud L. Expanding wire stents in benign tracheobronchial disease: indications and complications. Ann Thorac Surg 1992;54:937-40.

14. Acuff TE, Mack MJ, Ryan WH. Simplified placement of a silicone tracheal Y stent. Ann Thorac Surg 1994;57:496-7.

15. Ramsey ER, Beamis JF. Laser bronchoscopy. Clin Chest Med 1995; 16:415-25.

16. Wood DE, Vallières E. Tracheobronchial resection and reconstruction. Arch Surg 1997;132:850-7.

17. Alfaro J, Varela G, de-Miguel E, de Nicolas JLM. Successful management of a tracheo-innominate fistula following placement of a wire self expandable tracheal Gianturco stent. Eur J Cardiothorac Surg 1993;7:615-6.

18. Wood DE. Tracheal tumors. In: Wood DE, Thomas CR, editors. Mediastinal tumors. Berlin: Springer-Verlag; 1995. p. 87-93.

19. Grillo HC, Donahue DM, Mathisen DJ, Wain JC, Wright CD.
Postintubation tracheal stenosis: treatment and results. J Thorac Cardiovasc Surg 1995;109:486-93.

20. Chang LF, Horvath J, Peyton W, Ling SS. High dose rate afterloading intraluminal brachytherapy in malignant airway obstruction of lung cancer. Int J Radiat Oncol Biol Phys 1994;28:589-96.

21. Hayata Y, Kato H, Konaka C, et al. Photodynamic therapy in early stage lung cancer. Lung Cancer 1993;9:287-94.

22. LoCicero J, Metzdorff M, Almgren C. Photodynamic therapy in the palliation of late stage obstructing non-small cell lung cancer. Chest 1990;98:97-100.

\section{Discussion}

Dr Richard G. Fosburg (Del Mar, Calif). You mention that your facility has become a referral center, and I think this is insightful into what might be considered a center of excellent patient care. The opportunities for disaster are very high in attempting to correct these problems without adequate experience and a team approach involving the anesthesiologist, the surgeon, and the diagnostic evaluations that are done to identify the problems. There is a similar endoscopic center in San Diego, and most of the patients in our community are referred to that center.

One of the things that stands out in your report is the experience with stents. Your slides indicate that you have placed close to 80 stents. What has been the rate of retention and extraction, and what has been the usefulness of the stenting process itself? Could you address your remarks to those with anastomotic strictures?

Dr Stephens. Regarding the anastomotic strictures, during the time in which this study was conducted, amassing our lung transplantation, tracheal resection, and bronchoplastic resections, there were 120 anastomoses potentially at risk for stenosis. From that number, there were a total of 9 complications, 5 of which were minor, such as suture granuloma or a small amount of bleeding. There were 4 major complications, specifically tight strictures that required stenting and dilation. We are not displeased with that complication rate $(3.3 \%)$ for our anastomoses. We believe that there is a potential application for stenting. If these cases are recognized early during the phase of wound healing, before firm circumferential scar has occurred, there is a possibility that stent placement may permit tracheobronchial remodeling and the avoidance of the need for a stent. Regarding stent placement in general, we believe that the stent should be reserved as a last resort for those stenoses that are not treatable without the placement of an artificial device. The reason for that belief is that stents have their own set of complications and are somewhat labor intensive devices to maintain. The ridges on the stent are turbulence generators. They act to retain secretions. If we do not choose exactly the correct size of stent or if the airway becomes less edematous and allows the stent to loosen, it can migrate and become repositioned. Once in awhile, the patient will actually cough the stent out. If that happens, it is usually because the stent is not needed anymore, so that is not always an adverse outcome. However, the rate of stent migration and the rate of stent manipulation is about $5 \%$ in both the malignant and the benign groups. 
Dr Fosburg. You use of the terms "malignant" and "benign." I have a suggestion. Perhaps you would consider the idea that these conditions all are malignant in nature because they place the patient at significant risk. Because of our litigious society, it might be better to label them as neoplastic and non-neoplastic so that we do not attempt to imply that this is a benign condition or one that is not fraught with problems.

Dr Arthur Thomas (San Francisco, Calif). I was a bit surprised at the number of strictures you had from tracheostomy stomas, tracheomalacia, and benign cicatricial strictures. We at San Francisco General Hospital almost never see these complications because of low-pressure cuff tubes. We are very comfortable in leaving a tracheal tube in place in patients in the intensive care unit for 2 to 3 months before conversion to tracheostomy. Do you have any thoughts on why you have so many of these, or are they referral cases?

Dr Stephens. They have been accrued from a fairly wide referral network. Because of our location in Seattle, our referral network covers parts of Washington, Alaska, Montana, and Idaho. The numbers surprised us because they were larger than we had expected. However, given the fact that once those problems are detected they seem to be sent our way for management, we are not too surprised. Some of the causes are more surprising than the numbers themselves. For example, one of the more interesting cases was that of a 15-yearold girl who was in a pedestrian-versus-motorcycle crash as an infant and apparently had complete disruption of the left main-stem bronchus at that time. She was essentially living with a functional pneumonectomy for several years with a chronic cough until she was referred to us. A ventilation/perfusion scan showed almost zero ventilation on the affected side. After undergoing bronchoscopic dilation, she underwent a curative resection and had near equilibration of ventilation and perfusion on the two sides. I think that is a testimony to the oddity of some of the benign stenoses that we see. Included in the miscellaneous category of benign stenoses were such things as a near-obstructing pyogenic granuloma, which our pathologists had a very hard time identifying anywhere else in the literature, and tuberculous lymphadenopathy of such severity that it was compressing the carina and the right main-stem bronchus, as well as some of the more distal airways. I think the prominence of the benign stenoses that you aptly pointed out is due partially to my lumping them together under a miscellaneous category for the purposes of brevity.

\section{Online-www.aats.org}

Now you can get The Journal of Thoracic and Cardiovascular Surgery online. The Journal online brings you faster delivery time, easy searching of current and back issues, links to PubMed, AATS, WTSA, and other important sites, and more. Visit the Journal online today.

\section{Receive tables of contents by e-mail}

To receive the tables of contents by e-mail, sign up through our Web site at http://www.mosby.com/jtcvs. Choose E-mail Notification. Simply type your e-mail address in the box and click the Subscription button.

Alternatively, you may send an e-mail message to majordomo@mosby.com. Leave the subject line blank and type the following as the body of your message: subscribe jtcvs_toc.

You will receive an e-mail to confirm that you have been added to the mailing list. Note that table of contents e-mails will be sent out when a new issue is posted to the Web. 